\title{
Seleção de pontos-objeto visando o monitoramento de estruturas a partir da propagação de erros por simulação
}

\author{
Selection of object-points for structure monitoring from propagation of \\ errors by simulation
}

Lívia Faria Sampaio ${ }^{1}$

Regiane Dalazoana ${ }^{2}$

Luis Augusto Koenig Veiga ${ }^{3}$

Recebido em abril de 2019.

Aprovado em junho de 2019.

\begin{abstract}
RESUMO
Erros são inerentes ao processo de medida. Na prática, uma série de procedimentos são efetuados de forma a ter maior controle sobre estes erros. Trabalhos que envolvem levantamentos de campo visando o monitoramento de estruturas também apresentam incertezas associadas à instrumentação utilizada, tipo de mensurações e geometria da rede de monitoramento, esta última em função da localização da estação ocupada com o equipamento de medida em relação aos pontos que serão monitorados (pontos-objeto). Neste trabalho, são realizadas simulações visando prever o comportamento dos erros propagados em um levantamento, considerando a utilização do método de estação livre para orientação da estação total e do método de irradiação tridimensional para o monitoramento de grandes estruturas civis, como barragens. Busca-se a representação tridimensional dos erros propagados sobre a estrutura, de maneira a dar suporte aos técnicos de segurança de barragens na escolha e seleção de pontos-objeto de forma que, ao mesmo tempo que atendam à localização espacial requerida pela equipe de monitoramento civil, também apresente os menores valores propagados de variância. Com a metodologia da estação livre e irradiação tridimensional foram realizadas simulações em uma área teste considerando diferentes posições para implantação dos pontos de apoio para orientação da estação total, e diferentes posições para a estação total utilizada no levantamento. Como resultado da pesquisa foi desenvolvido um plugin, em software livre, disponibilizado ao usuário, que permite o cálculo dos valores propagados no
\end{abstract}

${ }^{1}$ Universidade Federal do Paraná. Programa de Pós-graduação em Ciências Geodésicas, Brasil. E-mail: liviasampaio@ufpr.br, regiane@ufpr.br, kngveiga@gmail.com. 
modelo da estrutura avaliada. Dessa forma foi possível analisar diferentes geometrias para o posicionamento da estação total.

PALAVRAS-CHAVE: Monitoramento de estruturas. Propagação de Erros. Simulações.

\begin{abstract}
Errors are inherent in the measurement process. In practice, a number of procedures are performed in order to have greater control over these errors. The surveying activities with the goal of monitoring structures present uncertainties due to the instrumentation and the geometrical configuration of the monitoring network, the last one depending on the position of the total station in relation to the points that will be monitored (object-points). In this paper, simulations are performed to predict the behavior of these propagated errors in the surveying, considering the use of the free station method for total station orientation and the three-dimensional polar method for the monitoring of large civil structures, such as dams. The threedimensional modeling and representation of the errors propagated on the structure aims to support dams security technicians in the selection of object-points so that it is possible to use the spatial location required by the team of civil monitoring and also it is possible to presents the lowest values of propagated variance. With the methodology of free station and threedimensional polar surveying, simulations were performed in a test area considering different positions for implantation of the reference points used in the total station orientation, and different positions for set up the total station used in the survey. As one of the results of the research a plugin was developed, in free software that is available to the user, which allows the calculation of the values propagated in the model of the evaluated structure. Thus, it was possible to analyze different geometries for the positioning of the total station.
\end{abstract}

KEYWORDS: Monitoring Structures. Error Propagation. Simulations.

$$
* * *
$$

\title{
Introdução
}

Atualmente as técnicas de levantamentos geodésicos são utilizadas largamente no âmbito de monitoramento de estruturas. A execução de levantamentos de campo com esse objetivo, normalmente passa pelas etapas de: planejamento; execução; realização de cálculos (ajustamento) e divulgação dos resultados e sua qualidade (KUANG, 1996). Na etapa de planejamento, também conhecida como pré-análise, é necessário que seja realizado um 
estudo prévio da área de estudo, e que sejam definidos alguns requisitos para a atividade de levantamento, como por exemplo, a precisão a ser alcançada, o instrumental a ser utilizado, as observações a serem realizadas, a geometria do problema a ser resolvido, o custo total do levantamento, a confiabilidade e a forma de controle dos erros.

Os levantamentos de campo visando o monitoramento de um objeto ou estrutura normalmente implicam em determinar uma grandeza que esteja correlacionada ao mesmo, como um comprimento ou coordenadas de um ou vários pontos deste objeto (pontos-objeto). Isto é feito através de medições diretas ou indiretas. Em ambas as situações, caso estas medições estejam eivadas de erro (e teoricamente toda observação apresenta erros), estes de alguma maneira irão afetar as grandezas desejadas/determinadas, uma vez que serão propagados para as mesmas. O estudo disto é chamado de propagação de erros. Mas é importante lembrar que, quando se trabalha com a propagação, assume-se que não existem erros sistemáticos e grosseiros na amostra e apenas erros aleatórios estão presentes (GHILANI, 2017).

Na simulação, o problema de propagação de erros tem como finalidade determinar a precisão das medidas que irá satisfazer um limite de tolerância específico para uma quantidade desconhecida a ser determinada. A simulação pode mostrar que as exigências da precisão das medidas estão abaixo ou além da capacidade exigida. Se a exigência for além das capacidades de medida, o cliente deve ser informado que o limite de tolerância especificado pode não ser alcançado (OGUNDARE, 2015).

O método que tem sido muito empregado para a orientação da estação total em um trabalho de levantamento é o método de estação livre, também conhecido na literatura como método de ressecção, ou interseção à ré. Este método vem sendo utilizado largamente no âmbito de monitoramento para a detecção de deslocamentos de estruturas (SHULTS e ROSHCHYN, 2016).

Ao realizar um levantamento com estação total, um profissional não necessariamente ocupa um ponto de uma rede de referência pré-estabelecida, 
neste caso, pode-se então realizar visadas a dois ou mais pontos préestabelecidos e empregar o método de estação livre para determinar as coordenadas do ponto ocupado. Este método, como o termo sugere, permite ao profissional escolher o melhor local para posicionar a estação total e determinar a sua distância e direção em relação a dois ou mais pontos (KLEIN et al. 2017). Esta característica de posicionamento da estação, otimiza o trabalho de campo e acaba tornando o uso deste método ainda mais viável para levantamentos visando o monitoramento de estruturas.

No âmbito dos levantamentos geodésicos alguns métodos são utilizados para o monitoramento de estruturas, entre eles estão o nivelamento geométrico de primeira ordem, levantamento gravimétrico, posicionamento GNSS (Global Navigation Satellite System) e irradiação tridimensional. Cada um desses métodos para levantamentos utiliza equipamentos, técnicas e procedimentos de campo distintos. Logo, para cada tipo de levantamento a modelagem do problema para determinação dos erros propagados será diferente. A partir do modelo matemático utilizado para cada um desses métodos, pode-se aplicar a lei de propagação das covariâncias para determinação dos erros propagados nesse levantamento.

Com isso, neste trabalho, avalia-se o erro propagado em pontos-objeto localizados na estrutura a ser monitorada, que neste caso é uma barragem, considerando o modelo matemático do método de levantamento de irradiação tridimensional e os erros devidos à orientação da estação total utilizando o método de estação livre, testando quais as geometrias possíveis e quais resultam nos menores valores dos erros propagados para os pontos-objeto. Além disto, busca representar tridimensionalmente sobre o modelo da estrutura estudada os valores propagados para as variâncias (e consequentemente os desvios-padrão) dos ponto-objetos definidos na etapa da modelagem, servindo assim de ferramenta para o planejamento e seleção de pontos a serem monitorados, usando como um dos critérios a menor variância para as coordenadas dos pontos. 


\section{Lei de Propagação das Covariâncias}

A lei de propagação das covariâncias para equações lineares e não lineares, pode ser escrita da seguinte forma (GHILANI, 2017):

$$
\sum_{z z}=A \sum A^{T}
$$

onde $\sum_{z z}$ é a matriz de covariância da função $Z$, e $\sum$ é a matriz de variância e covariância das observações. Para um conjunto não linear de equações que são linearizadas utilizando o teorema de Taylor, o coeficiente matricial A é chamado de matriz Jacobiana. Isso é, uma matriz das derivadas parciais em relação às incógnitas.

A função $\mathrm{Z}$ se refere a um modelo matemático escrito em função de uma série de observações. Em geral são fornecidas as observações e suas respectivas variâncias (ou o próprio valor do desvio-padrão), permitindo assim a montagem da matriz de variância e covariância das observações. E é requerida/calculada a matriz de variância e covariância das incógnitas $\left(\sum_{z z}\right)$.

Neste trabalho, como foi utilizada a lei de propagação das covariâncias para a realização de uma pré-análise, as precisões das coordenadas dos pontos-objeto são obtidas a partir de uma estimativa a priori das observações. Isso ocorre pois como a lei de propagação das covariâncias será aplicada para à função correspondente ao modelo matemático do método de irradiação tridimensional (que é um modelo não linear) logo, são necessários valores para as observações, precisões destas observações e precisão das coordenadas do ponto ocupado com a estação total. 


\section{Método de Estação Livre e Método de Irradiação Tridimensional}

\subsection{Método de estação livre}

O método de estação livre consiste em estacionar um instrumento topográfico sobre o ponto que se deseja determinar as coordenadas e realizar observações de direções a três pontos de coordenadas conhecidas. As coordenadas do ponto da estação são determinadas sem a necessidade da medição de distância entre os pontos observados (SILVA e SEGANTINI, 2015).

Pode-se também resolver o método de estação livre utilizando o Método dos Mínimos Quadrados (MMQ). Para aplicar o MMQ com a finalidade de estimar o valor dos parâmetros, utiliza-se o Método Paramétrico, em que os valores observados ajustados podem ser expressos como uma função dos parâmetros ajustados.

Para a aplicação desse método é necessário montar as equações de observação, que compõem o modelo matemático relacionando parâmetros e observações. Para facilitar o entendimento do modelo matemático adotado para o método de estação livre, observa-se a Figura 1, onde tem-se que:

$X_{A}, Y_{A}, Z_{A}$ : coordenadas do ponto da estação de controle A;

$X_{B}, Y_{B}, Z_{B}$ : coordenadas do ponto da estação de controle B;

$X_{C}, Y_{C}, Z_{C}$ : coordenadas do ponto da estação de controle C;

$X_{E}, Y_{E}, Z_{E}$ : coordenadas do ponto da estação livre E. 
Figura 1 - Representação do método de estação livre

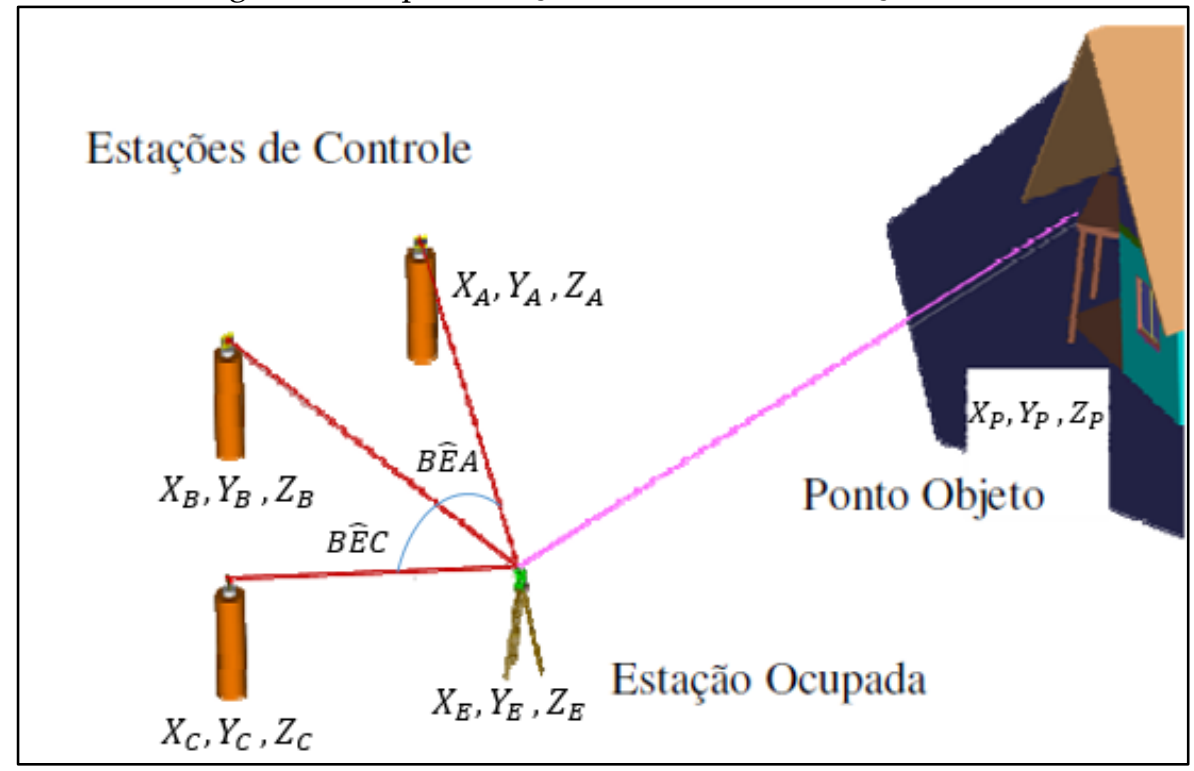

Fonte: Adaptado de Miranda e Veiga (2010).

As equações de observação utilizadas foram desenvolvidas para o cálculo das distâncias, ângulos horizontais e ângulos zenitais (calculadas entre as coordenadas do ponto da estação livre e as coordenadas dos pontos de controle) que compõem o modelo matemático relacionando parâmetros e observações.

\subsection{Método de irradiação tridimensional}

Também conhecido como método polar, a irradiação tridimensional consiste na determinação de coordenadas espaciais baseada na medição de direções horizontais, ângulos verticais e distâncias inclinadas entre a estação e o ponto a ser medido (SILVA e SEGANTINI, 2015). São consideradas as medidas realizadas entre: a) o ponto correspondente à posição da estação ocupada com o equipamento de medida, e b) os pontos com as coordenadas a serem monitoradas.

No contexto da pré-análise, como são necessários valores estimados para as observações, estes podem ser calculados a partir de valores 
aproximados para as coordenadas, medidas em um referencial local, da seguinte forma:

a) Cálculo da distância espacial entre o ponto $E$ (ponto correspondente à posição da estação total) e os pontos $P i$ (pontos a serem monitorados):

$$
d_{E P i}=\sqrt{\left(X_{P i}-X_{E}\right)^{2}+\left(Y_{P i}-Y_{E}\right)^{2}+\left(Z_{P i}-Z_{E}\right)^{2}}
$$

onde:

$X_{P i}, Y_{P i}, Z_{P i}:$ coordenadas do i-ésimo ponto a ser monitorado;

$X_{E}, Y_{E}, Z_{E}$ : coordenadas do ponto ocupado pela estação total;

$d_{P E i}$ : distância entre a estação total e o ponto a ser monitorado;

b) Cálculo do ângulo zenital entre $E$ e $P i$ :

$$
V_{E P i}=\arccos \frac{Z_{P i}-Z_{E}}{\sqrt{\left(X_{P i}-X_{E}\right)^{2}+\left(Y_{P i}-Y_{E}\right)^{2}+\left(Z_{P i}-Z_{E}\right)^{2}}}
$$

onde:

$V_{E P i}$ : ângulo zenital calculado entre a estação total e o ponto a ser monitorado $P i$.

c) Cálculo do azimute da direção formada entre $E$ e $P i$ :

$$
A_{E P i}=\operatorname{arctg} \frac{X_{P i}-X_{E}}{Y_{P i}-Y_{E}}
$$

onde:

$A_{E P i}$ : azimute calculado para a direção formada entre o ponto ocupado com a estação total e o ponto a ser monitorado; 
d) Cálculo dos ângulos horizontais:

$$
H_{E P i+1}=A_{E P i+1}-A_{E P i}
$$

onde:

$H_{E P i+1}$ : ângulo horizontal formado entre duas direções $E P i+1$ e $E P i$.

A representação das coordenadas é realizada através de um sistema com três eixos ortogonais entre si, formando um sistema de coordenadas tridimensionais ortogonais (Figura 2). Neste caso, considera-se o centro do sistema de coordenadas como sendo coincidente com o ponto Cardã.

Figura 2 - Representação do método de irradiação tridimensional

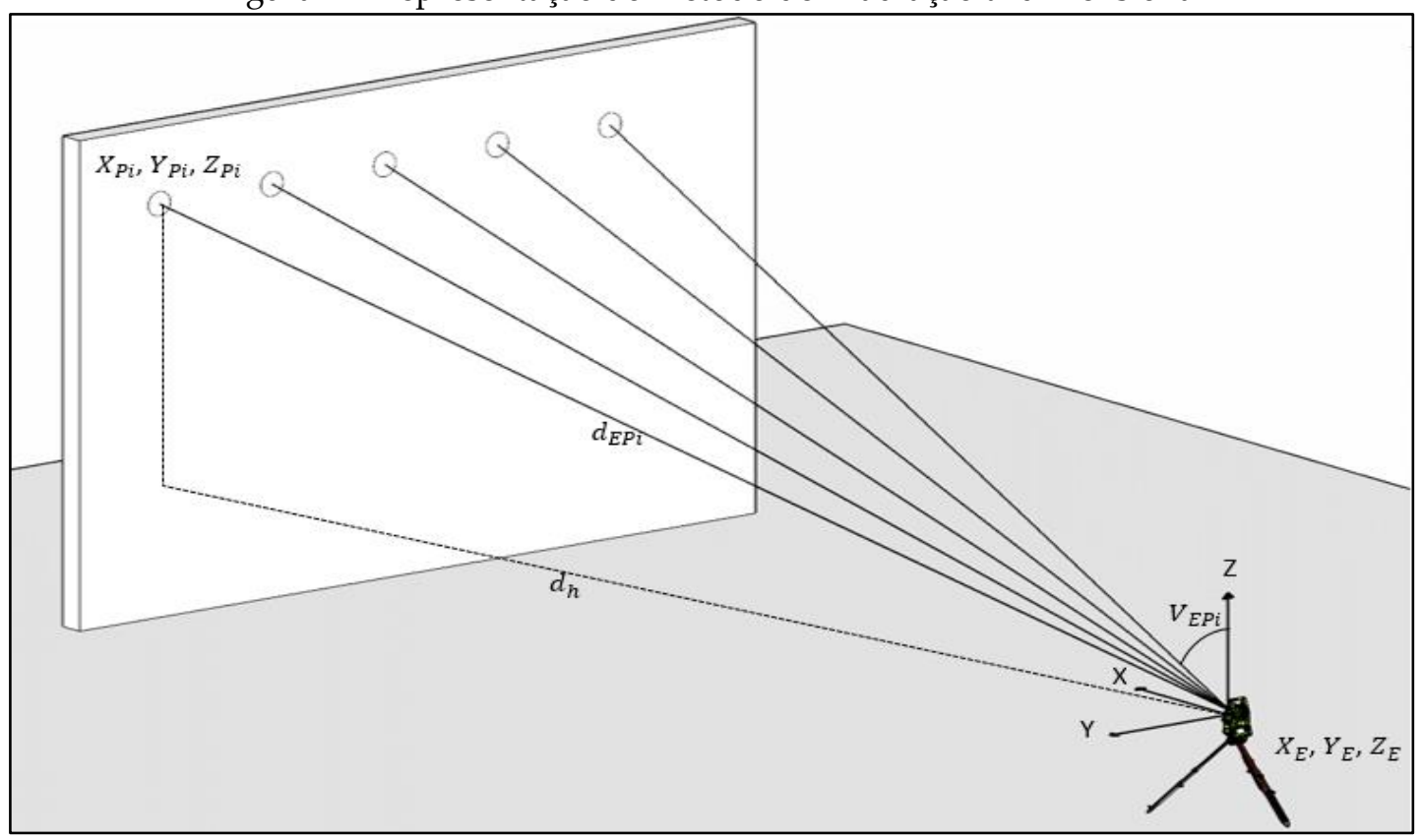

Fonte: Elaborada pelos autores.

Considerando o sistema de coordenadas representado na Figura 2, tem-se que as coordenadas dos pontos monitorados são obtidas a partir das equações de posicionamento neste sistema, que são:

$$
X_{P i}=d_{E P i} \operatorname{sen} V_{E P i} \operatorname{sen} A_{E P i}+X_{E}
$$




$$
\begin{gathered}
Y_{P i}=d_{E P i} \operatorname{sen} V_{E P i} \cos A_{E P i}+Y_{E} \\
Z_{P i}=d_{E P i} \cos V_{E P i}+Z_{E}
\end{gathered}
$$

onde:

$X_{P i}, Y_{P i}, Z_{P i}:$ coordenadas dos pontos a serem monitorados;

Os demais elementos das equações já foram explicados anteriormente.

A altura do instrumento não foi considerada na definição destas equações, uma vez que se considerou que a estação foi orientada nesta posição a partir do método de estação livre.

\section{Materiais e Métodos}

\subsection{Materiais}

Para o desenvolvimento deste trabalho de pesquisa, foram utilizados softwares que permitiram a elaboração de rotinas de programação para o cálculo iterativo das propagações de erro, além de facilitarem a manipulação e visualização dos dados, sendo estes: QGIS versão 2.14.14- software gratuito e de código aberto para Sistemas de Informações Geográficas; PgAdmin III plataforma de administração e desenvolvimento de código aberto para o PostgreSQL, que é um banco de dados também de código aberto; Python 3.0 Python é uma linguagem de programação que permite trabalhar e integrar sistemas com mais eficiência. É desenvolvido sob uma licença de código aberto; QTDesigner - ferramenta utilizada para projetar e construir interfaces gráficas de usuário (GUIs); AUTOCAD 2018 - Versão estudante; ParaView 5.5 - $R C 4$ - software livre que permite a visualização e análise de dados. Nesse programa é possível criar visualizações para análises de dados qualitativas e quantitativas. A exploração de dados pode ser feita interativamente em 3D ou a partir de comandos de programação. 
Também foram utilizados ortofotos e um modelo 3D da área de estudo para facilitar o reconhecimento da mesma.

\subsection{Estudo de caso}

Para o estudo de caso apresentado neste trabalho utilizou-se informações referentes a Usina Hidrelétrica de Mauá, localizada no rio Tibagi, entre os Municípios de Ortigueira e Telêmaco Borba, no estado do Paraná.

Alguns trabalhos desenvolvidos na Universidade Federal do Paraná (UFPR) realizaram atividades ligadas ao monitoramento da estrutura da barragem de Mauá, como por exemplo, os trabalhos realizados por Faggion, Nadal e Veiga (2012), Siguel (2013), Cruz (2015) e Gonçalves (2018). A partir desses estudos, foram disponibilizados alguns dados referentes a essa área, dentre eles um arquivo contendo a modelagem da estrutura em 3D (Figura $3)$.

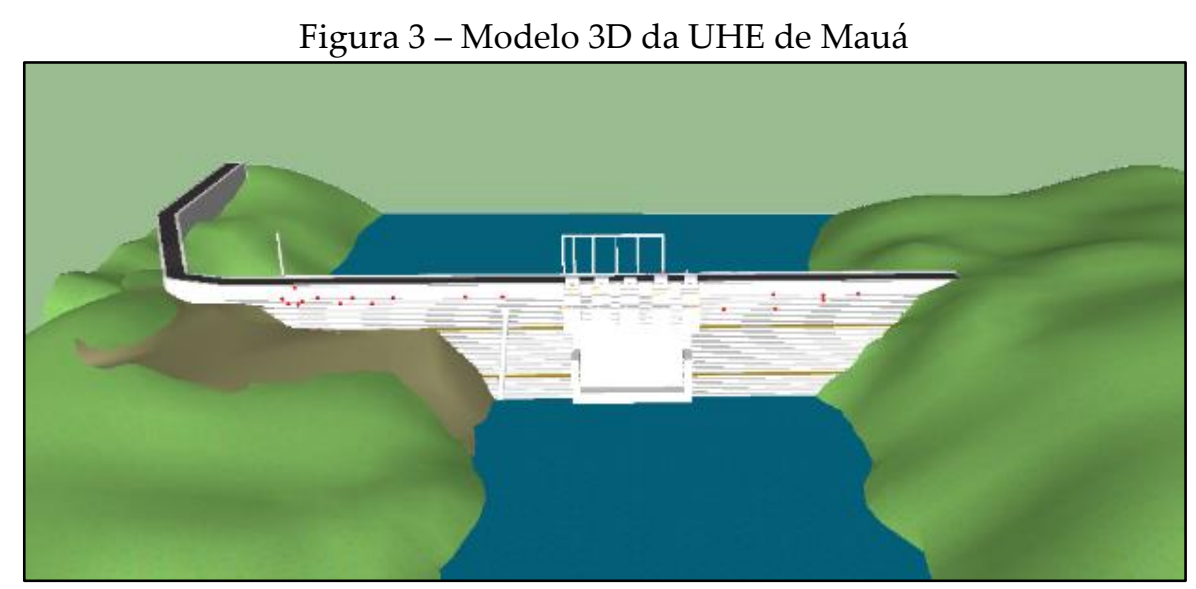

Fonte: Gonçalves (2018).

Com base nesse modelo 3D, foi simulada uma nuvem de pontos contornando toda a estrutura da barragem, a fim de obter coordenadas tridimensionais aproximadas para o conjunto de pontos. A partir destas 
coordenadas foi possível estimar as observações (de direções e distâncias) necessárias a realização da propagação de erros. Esses pontos foram criados no software Autocad utilizando uma ferramenta de criação de pontos. Os pontos foram distribuídos na fachada a jusante da barragem, e, ao todo, foram criados aproximadamente 5000 pontos (Figura 4).

Figura 4 - Nuvem de Pontos

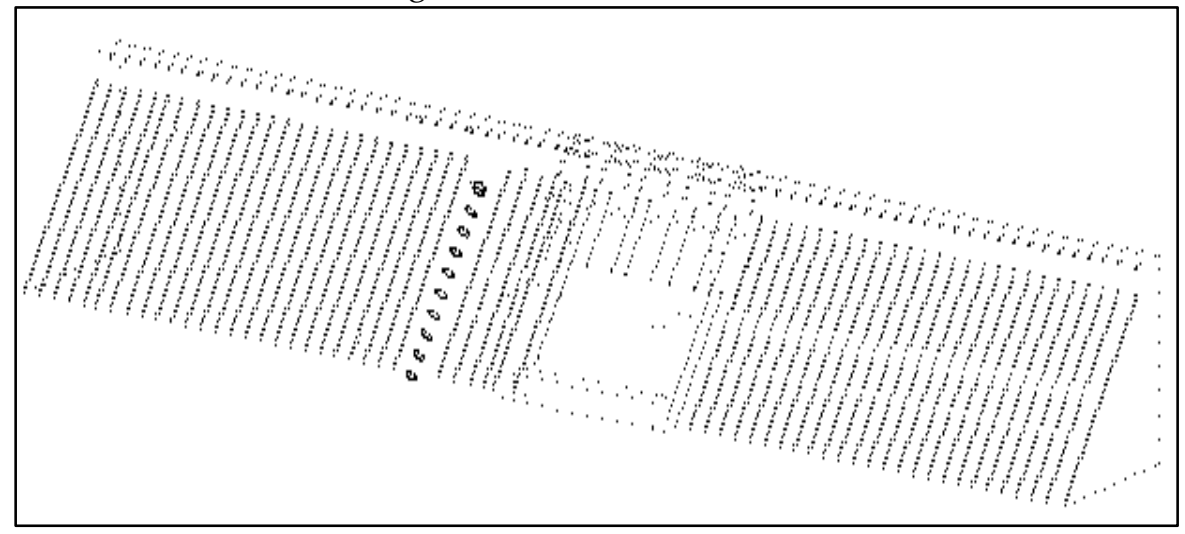

Fonte: Elaborada pelos autores.

3.3 Desenvolvimento da aplicação geoespacial utilizando python

Com o objetivo de calcular de forma interativa o valor das precisões das coordenadas dos pontos-objeto, desenvolveu-se um plugin, no software livre QuantumGis, com uma rotina de programação que permite a realização destes cálculos. Para facilitar o entendimento desse plugin, elaborou-se um fluxograma (Figura 5) no qual são apresentados os dados de entrada, os dados de saída e as etapas da rotina de programação que foi desenvolvida. 
Figura 5 - Fluxograma

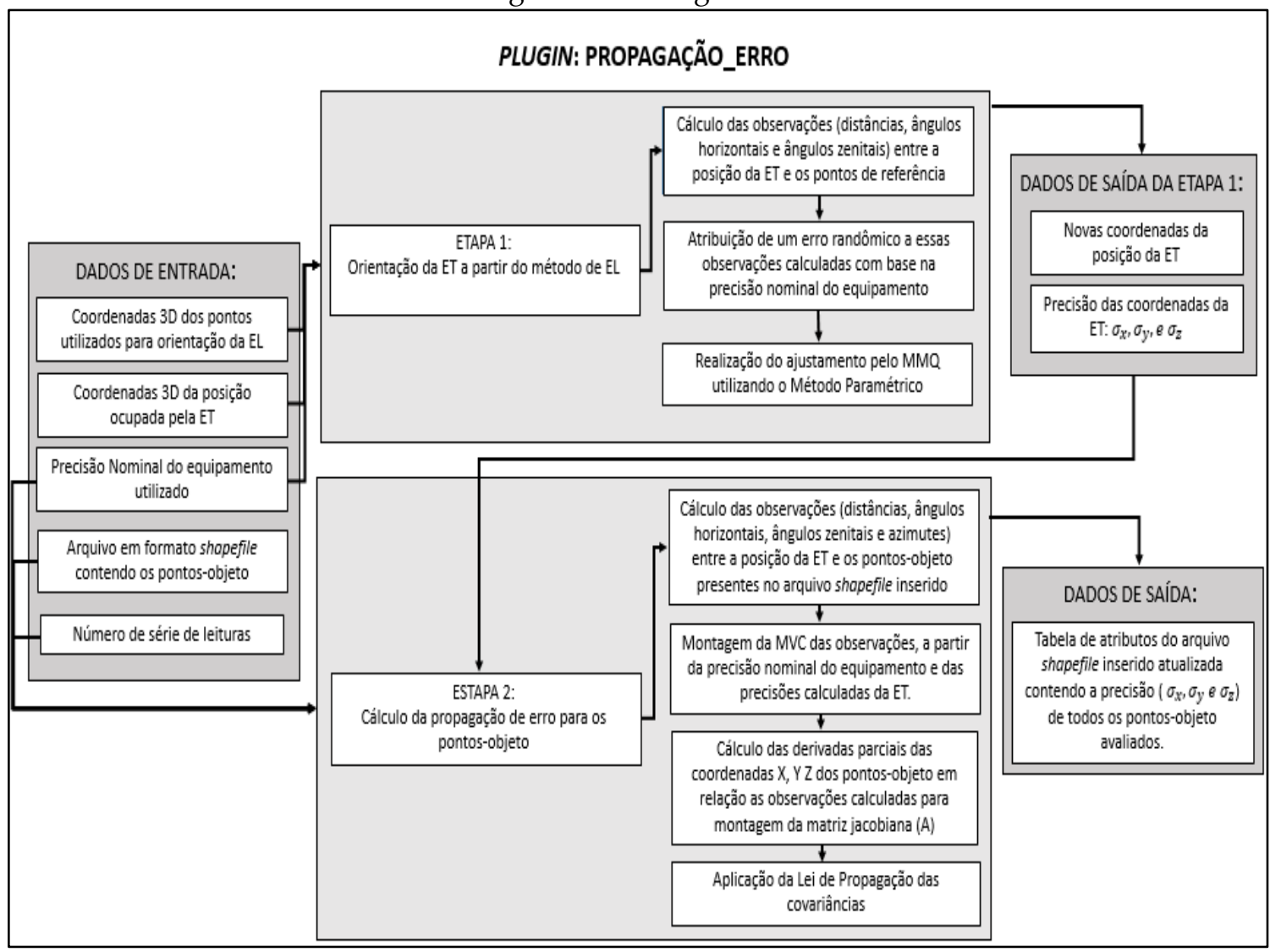

Fonte: Elaborada pelos autores.

No fluxograma apresentado, as abreviações ET e EL referem-se à estação total e estação livre respectivamente. Além da precisão nominal do equipamento, o usuário também deve inserir no plugin um arquivo no formato shapefile contendo as coordenadas aproximadas dos pontos que possivelmente serão monitorados. Essa extensão de arquivo armazena a posição, forma e atributos de feições geográficas, sendo estes atributos os valores dos desviospadrão calculados para cada ponto. Os dados são organizados em forma de tabela nesse formato de arquivo. Assim, no arquivo shapefile contendo os pontos da estrutura (barragem), foram criados campos na tabela de atributos para que os resultados obtidos com os cálculos de propagação de erros fossem armazenados nesses espaços. 
Como resultado o plugin atualiza a tabela de atributos dos pontos a serem monitorados, e devem constar nessa tabela os valores do $\sigma_{x}, \sigma_{y}, \sigma_{z}$ (precisões calculadas para as coordenadas $\mathrm{X}, \mathrm{Y}$ e Z dos pontos a serem monitorados).

3.3.1 Orientação da estação total pelo método de estação livre

No desenvolvimento das simulações optou-se pelo posicionamento do equipamento através do método de estação livre, com orientação da estação total a partir de três pontos conhecidos. Dessa forma consegue-se calcular a coordenada tridimensional do ponto ocupado pela estação total e sua precisão. É possível orientar a estação total somente com dois pontos conhecidos, porém a utilização de três pontos para essa orientação fornece uma abundância de observações, o que permite a realização do ajustamento de observações.

Como os dados utilizados são simulados neste trabalho, e não propriamente medidos, atribuiu-se um erro às observações. Esse erro foi determinado de forma aleatória, considerando o quanto se espera que pode variar uma observação medida devido à precisão nominal do equipamento. Utilizou-se então, para essa determinação, uma função pré-existente na linguagem Python denominada "np.random.standard_normal", que calcula um valor aleatório variando de 0 a 1 da distribuição normal. Esse valor foi multiplicado pelas precisões nominais do equipamento utilizado para a simulação e, com isso, obtiveram-se as observações com possíveis "erros" para a realização do ajustamento.

Para o ajustamento utilizou-se o método paramétrico, que permitiu o cálculo das coordenadas e das respectivas precisões do ponto ocupado pela estação total. O modelo matemático utilizado para este ajustamento envolveu as seguintes equações: para as distâncias entre os pontos de apoio e a estação total utilizou-se a equação (2); para os ângulos zenitais entre as direções dos 
pontos de apoio e a estação total, utilizou-se a equação (3), e os ângulos horizontais puderam ser estimados a partir das equações (4) e (5).

3.3.2 Aplicação da lei de propagação das covariâncias para o modelo matemático do método de irradiação $3 \mathrm{D}$

Após a estimativa da precisão das coordenadas da posição da estação total, realizou-se a simulação do monitoramento dos pontos-objeto.

Na pré-análise não se tem, a priori, as observações, por isso estas podem ser estimadas a partir das coordenadas aproximadas dos pontos-objeto e da estação total. Estas coordenadas aproximadas são obtidas a partir de alguma forma de reconhecimento prévio do terreno ou da área de estudo. Neste trabalho foram utilizadas as coordenadas dos pontos da nuvem de pontos criada da barragem; para, a partir das equações do método de irradiação tridimensional (equações 2, 3, 4 e 5), calcular os valores aproximados dos azimutes, dos ângulos verticais, das distâncias e das direções horizontais entre a posição da estação total e os possíveis pontos a serem monitorados.

Para a determinação da precisão (desvio-padrão) das coordenadas tridimensionais de pontos a serem monitorados na estrutura, aplicou-se a lei de propagação das covariâncias para o método de irradiação tridimensional. O modelo matemático é dado pelas equações de posicionamento (equações 6 , 7 e 8$)$.

$\mathrm{Na}$ fórmula da lei de propagação das covariâncias (equação 1) tem-se a matriz A que corresponde à matriz das derivadas parciais da função original (matriz jacobiana). No caso do modelo matemático utilizado, a matriz das derivadas parciais é determinada da seguinte forma: 


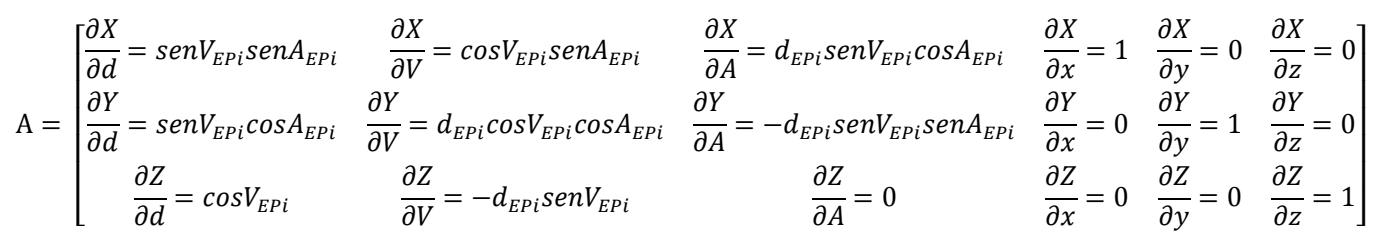

onde:

$\frac{\partial X}{\partial d}, \frac{\partial Y}{\partial d}, \frac{\partial Z}{\partial d}:$ são as derivadas parciais das coordenadas $\mathrm{X}, \mathrm{Y}$ e Z dos pontos monitorados em relação à distância;

$\frac{\partial X}{\partial V}, \frac{\partial Y}{\partial V}, \frac{\partial Z}{\partial V}$ : são as derivadas parciais das coordenadas $\mathrm{X}, \mathrm{Y}$ e Z dos pontos monitorados em relação ao ângulo zenital;

$\frac{\partial X}{\partial A}, \frac{\partial Y}{\partial A}, \frac{\partial Z}{\partial A}$ : são as derivadas parciais das coordenadas $\mathrm{X}, \mathrm{Y}$ e Z dos pontos monitorados em relação ao azimute;

$\frac{\partial X}{\partial x}, \frac{\partial Y}{\partial x}, \frac{\partial Z}{\partial x}$ : são as derivadas parciais das coordenadas $\mathrm{X}, \mathrm{Y}$ e Z dos pontos monitorados em relação à coordenada $\mathrm{X}$ do ponto ocupado pela estação total;

$\frac{\partial X}{\partial y}, \frac{\partial Y}{\partial y}, \frac{\partial Z}{\partial y}$ : são as derivadas parciais das coordenadas $\mathrm{X}, \mathrm{Y}$ e Z dos pontos monitorados em relação à coordenada Y do ponto ocupado pela estação total;

$\frac{\partial X}{\partial z}, \frac{\partial Y}{\partial z}, \frac{\partial Z}{\partial z}$ : são as derivadas parciais das coordenadas $\mathrm{X}, \mathrm{Y}$ e Z dos pontos monitorados em relação à coordenada $\mathrm{Z}$ do ponto ocupado pela estação total.

A fórmula da propagação de covariância (equação 1) apresenta também a matriz de variância-covariância (MVC) das observações estimadas, que para o caso da aplicação em questão será: 


$$
\Sigma=\left[\begin{array}{cccccc}
\sigma D^{2} & 0 & 0 & & & \\
0 & \sigma V^{2} & 0 & & & \\
0 & 0 & \sigma A^{2} & & & \\
& & & \sigma x^{2} & \sigma_{x_{e} y_{e}} & \sigma_{x_{e} z_{e}} \\
& & & \sigma_{y_{e} x_{e}} & \sigma y^{2} & \sigma_{y_{e} z_{e}} \\
& & & \sigma_{z_{e} x_{e}} & \sigma_{z_{e} y_{e}} & \sigma z^{2}
\end{array}\right]
$$

onde:

$\sigma D$ : é a precisão das distâncias;

$\sigma \mathrm{V}$ : é a precisão dos ângulos zenitais;

$\sigma A:$ é a precisão dos azimutes;

$\sigma x$ : é a precisão da coordenada x da posição da estação total;

бy: é a precisão da coordenada y da posição da estação total;

oz: é a precisão da coordenada z da posição da estação total;

$\sigma_{x_{e} y_{e}}$ : é a covariância calculada para as coordenadas $x_{e}$ e $y_{e}$.

Os valores utilizados para a montagem da MVC, descrita através da equação (10), foram obtidos da seguinte forma:

a) as precisões (ou as variâncias) e as covariâncias para as coordenadas $\mathrm{X}, \mathrm{Y}$ e Z das posições da estação total foram obtidas do ajustamento descrito no tópico anterior. b) a precisão da distância $\left(\sigma_{D}\right)$ foi obtida a partir da equação (11), descrita em Ghilani (2017), que permite estimar o erro de uma distância medida com um MED (medidor eletrônico de distância):

$$
\sigma_{D}=\sqrt{\frac{\sigma_{i}^{2}+\sigma_{t}^{2}+a^{2}+(D b p p m)^{2}}{n}}
$$

onde:

$\sigma_{D}$ : é o erro na distância medida;

$\sigma_{i}$ : é o erro de centragem do instrumento;

$\sigma_{t}$ : é o erro de centragem do refletor; 
a: é a parte constante da precisão do equipamento;

b: é a parte variável da precisão do equipamento;

D: é a distância medida;

$\mathrm{n}$ : número de séries de leituras executadas.

No caso do trabalho em questão, como foi utilizado o método de estação livre, o instrumento não é instalado em nenhum ponto conhecido, logo, considerou-se o erro de centragem do equipamento igual a zero. $\mathrm{O}$ erro de centragem do refletor também foi considerado igual a zero, uma vez que os pontos-objeto são fixos na barragem.

c) para o cálculo da precisão angular é necessário considerar que a diferença entre duas direções observadas permite a obtenção do ângulo. A precisão deste ângulo será função da precisão nominal angular da estação total. Normalmente para uma estação total, a precisão nominal angular é definida para um par de leituras conjugadas de uma direção. Assim, para uma estação total de 5", ao se efetuar a leitura da direção horizontal em um alvo em pontaria direta e depois em pontaria invertida, este valor indica que o desvio-padrão da média das duas leituras será de 5" (GIAA, 2002). Para um ângulo (diferença entre a leitura de duas direções efetuadas em pontaria direta e invertida) a precisão será de 7" (onde 7" $=5 " \sqrt{2}$ ). Os valores calculados para a precisão angular foram considerados na MVC para os valores de $\sigma \mathrm{V}$ e de $\sigma A$.

Após a montagem da matriz jacobiana (equação 09) e da MVC com os valores das precisões das distâncias, dos ângulos zenitais, dos azimutes, e das coordenadas do ponto ocupado com a estação total (equação 10), aplicou-se a lei de propagação das covariâncias a fim de obter os valores das precisões das coordenadas $\left(\sigma_{x}, \sigma_{y}, \sigma_{z}\right)$ de cada ponto-objeto. 


\subsection{Cenário dos testes aplicados}

A fim de avaliar diferentes cenários, definiram-se diferentes configurações possíveis de instalação da estação total para o monitoramento da estrutura, e também diferentes posições para instalação dos pontos que serão utilizados para orientação da estação no método de estação livre. A Figura 6 apresenta três cenários diferentes que foram avaliados por ocasião deste trabalho, onde os pontos EST 01, EST 02 e EST 03 ilustram possíveis locais para instalação da estação total e os pontos A, B e C ilustram a posição dos pontos utilizados na orientação do equipamento.

Figura 6 - Cenários Avaliados

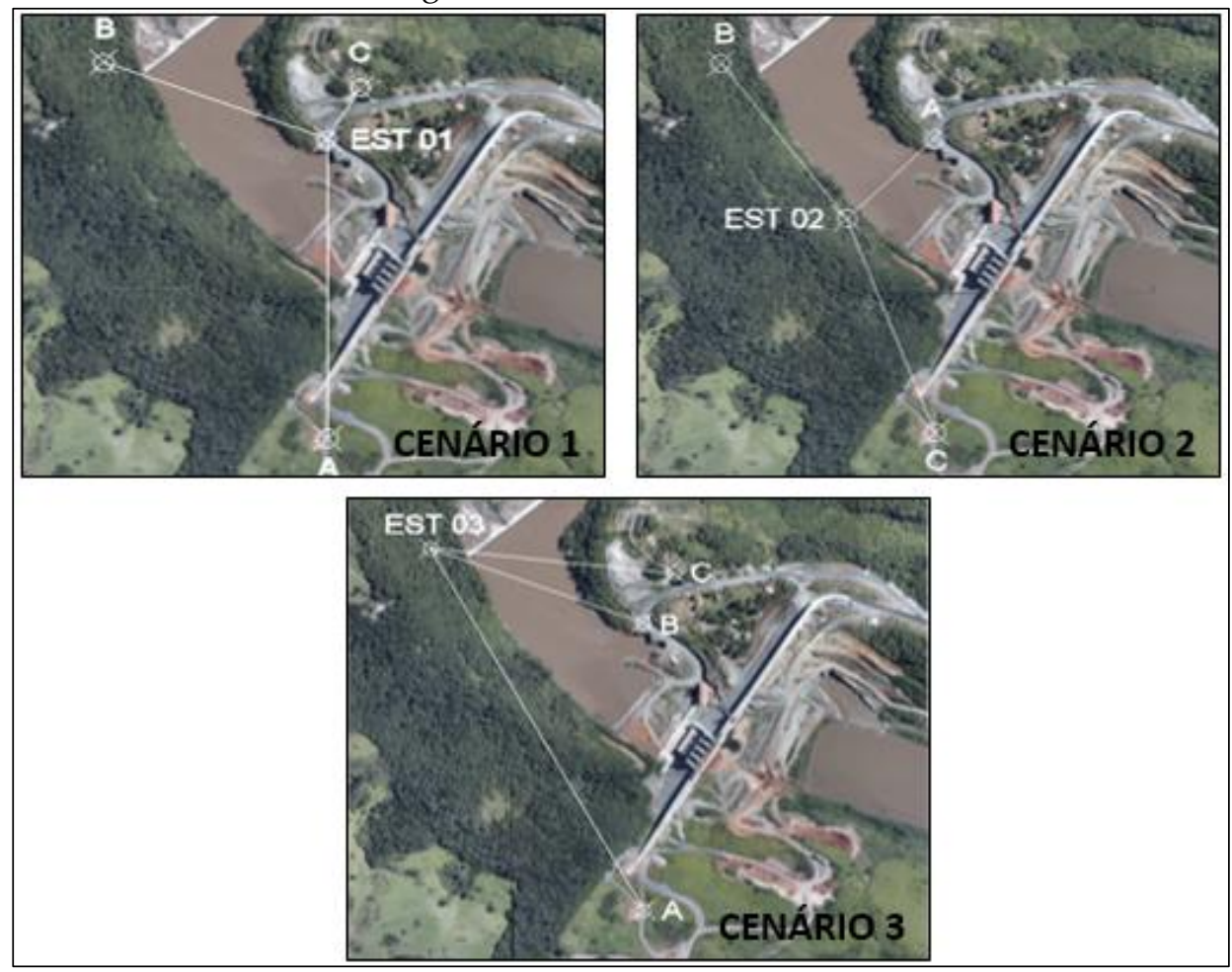

Fonte: Elaborada pelos autores. 


\section{Resultados e Análise dos Resultados}

\subsection{Plugin desenvolvido}

O plugin desenvolvido para o cálculo da propagação de erros apresenta na sua interface três janelas (Figura 7). Na primeira janela, com o nome de Orientacao_EstacaoLivre, o usuário deve entrar com as coordenadas dos três pontos que servirão de apoio para a orientação da estação total. $\mathrm{Na}$ segunda janela (Estacao_Livre), o usuário deve inserir as coordenadas de instalação da estação total, assim como a precisão nominal do equipamento utilizado. E na terceira janela, que se chama Pontos_monitorados, deve-se inserir o arquivo no formato shapefile, contendo os pontos que representam a estrutura que se deseja monitorar e o número de séries que se deseja executar em campo para o levantamento desses pontos.

Figura 7 - Plugin

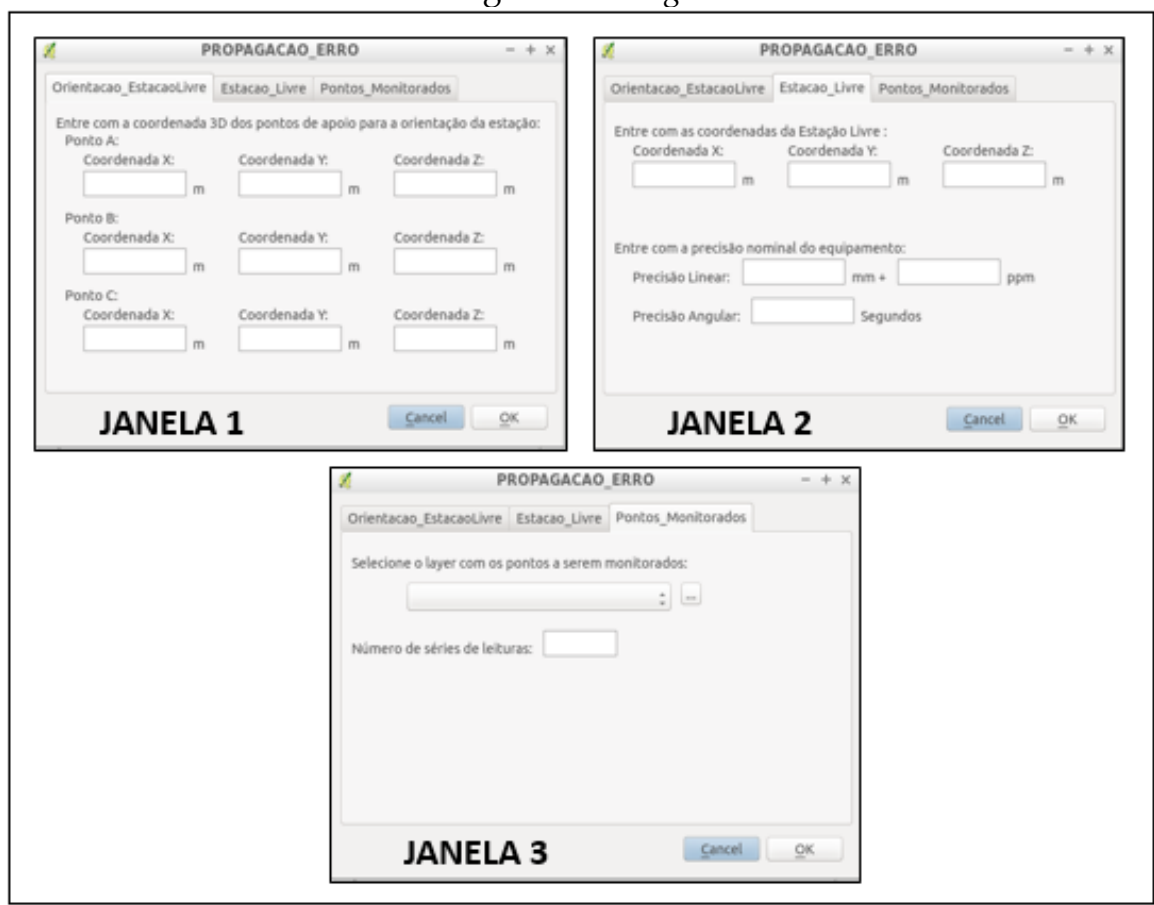

Fonte: Elaborada pelos autores. 
4.2 Avaliação da precisão dos pontos ocupados pela estação total nos diferentes cenários - método de estação livre

Para os testes aplicados, avaliou-se primeiramente os valores obtidos para a precisão das coordenadas dos pontos ocupados com a estação total. Os valores calculados dos desvios-padrão para cada teste podem ser observados na Tabela 1, onde tem-se que dpx corresponde aos valores do desvio-padrão calculados para a coordenada X da estação, dpy para a coordenada $\mathrm{Y}$ e dpz para a coordenada Z. A sigla dp3d corresponde ao valor da resultante do desvio-padrão calculado para as três componentes (X, Y e Z) e dp2d a resultante para as componentes $\mathrm{X}$ e $\mathrm{Y}$.

TABELA 1 - Valores dos desvios-padrão estimados para as estações

\begin{tabular}{c|c|c|c|c|c}
\hline CENÁRIO & $\mathbf{d p x} \mathbf{( m m})$ & $\mathbf{d p y} \mathbf{( m m})$ & $\mathbf{d p z} \mathbf{( m m})$ & $\mathbf{d p 2 d} \mathbf{( m m})$ & $\mathbf{d p 3 d}(\mathbf{m m})$ \\
\hline 1 & 1,1 & 1,1 & 0,6 & 1,5 & 1,7 \\
\hline 2 & 1,5 & 1,2 & 1,5 & 1,9 & 2,4 \\
\hline 3 & 1,9 & 3,0 & 2,3 & 3,6 & 4,3 \\
\hline
\end{tabular}

Fonte: Elaborada pelos autores.

Nos valores apresentados na Tabela 1 observa-se que o cenário 3 apresentou os maiores valores dos desvios-padrão calculados. Isso permite inferir que a geometria aplicada neste teste atribui maiores valores dos desvios-padrão se comparadas às demais geometrias avaliadas. $\mathrm{O}$ valor da resultante tridimensional para este cenário é cerca de $4,3 \mathrm{~mm}$, sendo maior se comparado ao cenário $1(1,7 \mathrm{~mm})$, e ao cenário $2(2,4 \mathrm{~mm})$.

4.3 Avaliação da precisão calculada para os pontos-objeto a partir das diferentes posições da estação - método de irradiação 3D

No primeiro teste foram inseridos no plugin os pontos da nuvem de pontos gerada a partir do modelo 3D da barragem (Figura 4), e calculou-se o desvio-padrão das coordenadas desses pontos com base na precisão nominal 
da estação total TS15 - precisão linear de $\pm(2 \mathrm{~mm}+2 \mathrm{ppm})$, e precisão angular de \pm 1 ". As simulações foram realizadas considerando uma (1X), três (3X) e oito (8X) séries de leituras, com a finalidade de verificar, quantitativamente, a evolução da melhora da precisão das coordenadas com o aumento do número de séries de leitura.

Na Figura 8 observam-se os valores calculados para os desvios-padrão resultantes do cenário 1 . Pode-se verificar a diferença entre os valores do desvio-padrão médio entre essas séries de leituras. Neste gráfico é possível observar que quanto maior o número de leituras melhor é a precisão obtida para os pontos-objeto. No caso da realização de uma série de leituras, o desviopadrão médio calculado foi de $3,9 \mathrm{~mm}$, a precisão média para três séries de leituras foi de 2,3 $\mathrm{mm}$ e para as oito séries de leituras foi de 1,4 $\mathrm{mm}$. Ou seja, a precisão melhorou cerca de $2,5 \mathrm{~mm}$ quando comparados os testes com 1 série e 8 séries de leituras.

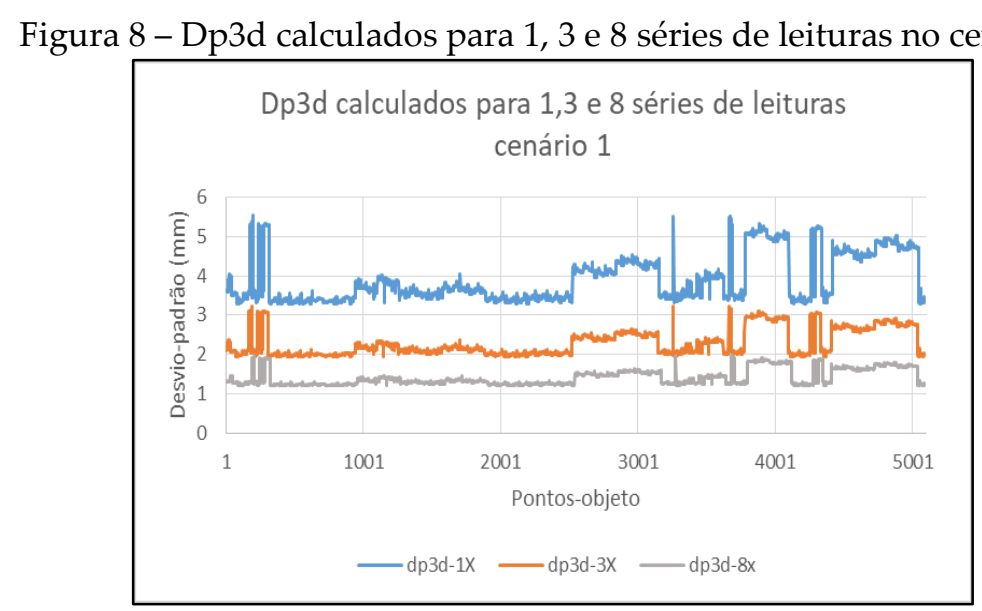

Fonte: Elaborada pelos autores.

A Figura 9 apresenta os valores médios dos desvios-padrão calculados nos cenários 1, 2 e 3 com apenas uma série de leituras. Observa-se que os menores valores encontrados foram os valores do cenário 1, onde a estação total se encontra mais próxima ao objeto monitorado (cerca de $220 \mathrm{~m}$ ). Também vele ressaltar que para o cenário 1 foram encontrados os menores 
valores dos desvios-padrão tridimensional avaliados em função da geometria adotada para a orientação da estação total neste cenário.

$\mathrm{Na}$ sequência, vem os valores calculados para o cenário 2 , no qual a estação se encontra a aproximadamente 260 metros da barragem. Os maiores valores foram os obtidos para o cenário 3, onde a estação está mais longe da barragem (605 m aproximadamente). Neste gráfico também é possível observar que as menores variações, em termos de amplitude, nos valores dos desvios-padrão estão no cenário 03, pois, apesar da estação estar localizada mais longe da barragem, é a estação que se encontra em uma posição mais centralizada em relação à barragem do que as outras duas.

Figura 9 - Dp3d calculados para os cenários 1, 2 e 3

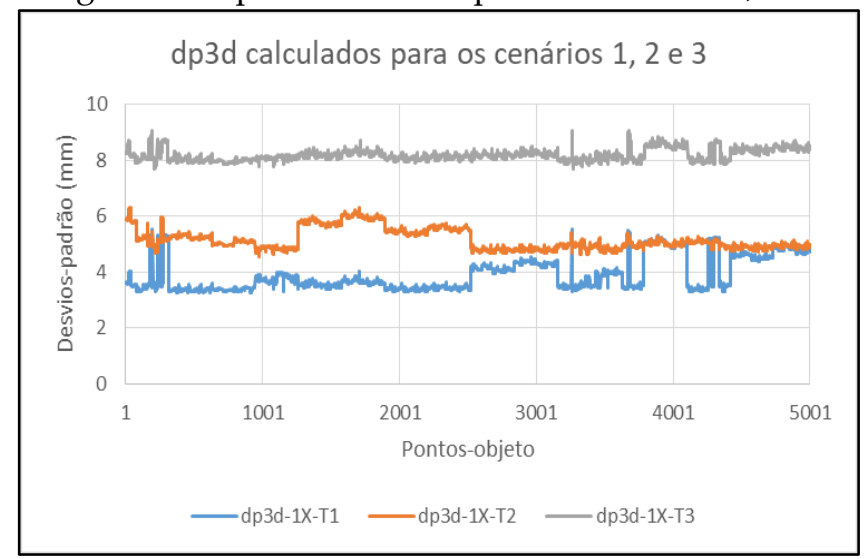

Fonte: Elaborada pelos autores.

A Figura 10 sumariza os gráficos gerados para cada cenário avaliado. Observa-se que no caso do cenário 1 , em que a posição da estação total está mais à esquerda da estrutura avaliada, os maiores valores dos desvios-padrão calculados estão do lado direito da estrutura, ou seja, do lado mais distante da estação. No cenário 2 a estação está localizada mais à direita e, devido a isso, os pontos com maiores valores de desvios-padrão estão à esquerda da estrutura. Para o caso do cenário 3, em que a estação está mais centralizada em relação à barragem, observa-se que os maiores valores dos desvios-padrão 
estão nos pontos localizados nas bordas direita e esquerda da estrutura. Para a geração da Figura 10, utilizou-se o software ParaView.

Figura 10 - Comparação entre os diferentes valores dos desvios-padrão dos cenários avaliados

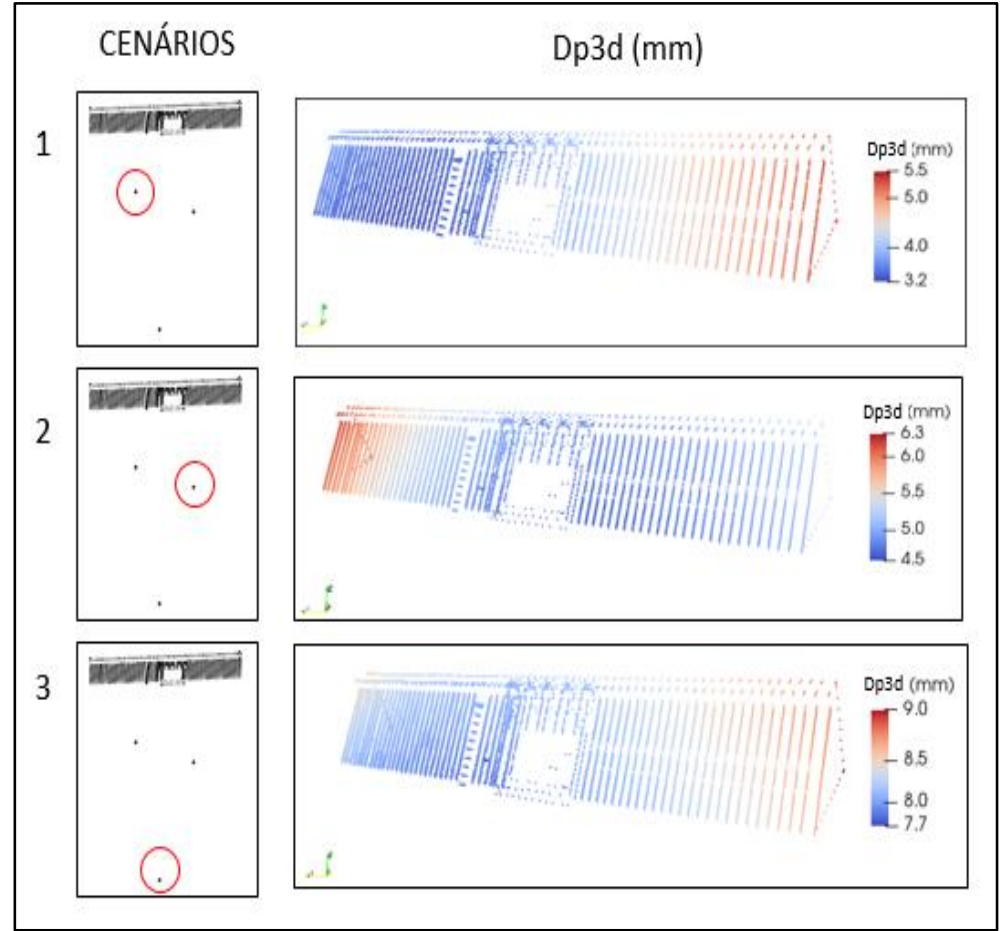

Fonte: Elaborada pelos autores.

\section{Considerações Finais e Conclusões}

A seleção de pontos-objeto em uma estrutura para fins de monitoramento é uma atividade que depende do tipo da estrutura e da configuração geométrica do levantamento, que engloba a disposição espacial dos pontos de referência, a posição e precisão do equipamento de medida e a geometria de observação. Neste trabalho apresentou-se um método que permite dar suporte à escolha da posição destes pontos com base na propagação das covariâncias, calculando as precisões esperadas na determinação das coordenadas do ponto na estrutura, com base em métodos de estação livre para o posicionamento do equipamento de medida, e 
irradiação 3D para o monitoramento dos pontos-objeto. Foi realizado um estudo de caso para a UHE de Mauá com diferentes cenários de configuração geométrica.

Neste trabalho foram simulados 3 cenários, com diferentes possibilidades de configuração para o posicionamento dos pontos de referência para a orientação da estação total a partir do método de estação livre. $\mathrm{O}$ cenário 3 foi o que apresentou os maiores valores dos desvios-padrão para as coordenadas da estação livre, sendo este com a pior geometria avaliada. O cenário que apresentou os melhores valores calculados foi o cenário 1. Devido a isso, foi possível avaliar a melhor posição de implantação dos pontos de controle utilizados para orientar a estação total.

Com a metodologia empregada foi possível também quantificar os erros propagados em uma estrutura, a partir da aplicação da propagação de erros utilizando o modelo matemático de irradiação tridimensional. A criação de uma nuvem de pontos representando a estrutura avaliada permitiu modelar a barragem, e a partir desses pontos foi possível calcular os valores dos erros propagados.

Para dar suporte aos cálculos matemáticos foi desenvolvido um plugin em linguagem de programação que permitiu o cálculo da propagação de erro para a nuvem de pontos que foi simulada para a UHE de Mauá. Esse plugin automatizou o processo dos cálculos, e permitiu que fossem realizadas as simulações de forma mais interativa. Assim, o uso do plugin permite a otimização do planejamento do levantamento de campo. Este aplicativo está disponível em: https://github.com/liviafariasampaio/PropagacaoErro.git.

Com os pontos-objeto representados em 3D, sobre o modelo da estrutura, foi possível analisar de forma gráfica as regiões em que foram apresentados os maiores e os menores valores dos desvios-padrão. Com isso, pode-se verificar qual a região mais propícia à implantação dos pontos-objeto, de forma que estes possam ser monitorados a partir de determinada posição 
da estação total, atingindo os menores valores dos erros propagados pela instrumentação e pelo método utilizado.

Esta representação gráfica dos resultados, foi feita com o software ParaView. Este permitiu representar de forma visual os valores calculados dos erros propagados na estrutura monitorada. O uso dessa ferramenta se mostrou importante para a visualização e compreensão dos resultados a partir da espacialização dos valores propagados sobre a estrutura.

Pode-se utilizar a metodologia aplicada para definir a posição de implantação de pontos-objeto para o monitoramento de estruturas de forma que a propagação de erros seja mínima (ou menor) para esses pontos. No entanto, é importante ressaltar que muitas vezes, dependendo da estrutura avaliada, as posições dos pontos-objeto devem ser realizadas em regiões previamente determinadas, uma vez que se sabe que nesta localização ocorre uma maior deformação da estrutura ou que nesta posição possa existir um ponto de interesse para um projeto. Portanto, nestes casos cabe à simulação indicar, principalmente, o local onde pode ser instalada a estação total com o objetivo de diminuir a influência dos erros e da geometria avaliada na determinação da sua posição.

Outro fator importante para atividades de monitoramento e de levantamentos em geral, é considerar a precisão exigida pelo projeto. Então, apesar de saber que a partir do monitoramento com a estação total instalada na posição mais próxima da barragem tem-se menores valores de desviopadrão, outras posições também podem ser consideradas para o monitoramento, se elas permitirem que sejam atingidas as precisões exigidas pelo cliente. Portanto, é importante ressaltar que são muitas as variáveis que devem ser consideradas para o planejamento de um levantamento. A préanálise pode fornecer várias soluções que atendem aos requisitos do cliente, cabe então verificar qual delas é a mais adequada. 


\section{Contribuição dos autores}

A pesquisa apresentada no artigo em questão foi realizada no período do mestrado acadêmico da autora principal, sob orientação dos demais coautores. Com isso, tem-se que a execução do projeto foi realizado pela autora Lívia Faria Sampaio, e os coautores, Regiane Dalazoana e Luis Augusto Koenig Veiga, realizaram as correções do trabalho, auxiliaram na ideia e na contribuição de materiais e referências para a execução dele.

\section{Referências}

CRUZ, W. Integração de dados de monitoramento de estruturas antrópicas. Estudo de caso: UHE Mauá. Tese de doutoramento. Universidade Federal do Paraná, Programa de Pós-Graduação em Ciências Geodésicas, Curitiba, 2015. 146p.

FAGGION, P. L.; NADAL, C. A.; VEIGA, L. A. K. Relatório Técnico 01-2012 Projeto PD6491-0227/2011 - Monitoramento Geodésico - Projeto e Implantação da rede de Auscultação Geodésica da UHE Mauá. Relatório Técnico, Universidade Federal do Paraná, Curitiba, 2012.

GHILANI, C. D. Adjustment computations: spatial data analysis. $6^{\mathrm{a}} \mathrm{ed}$. New Jersey: John Wiley \& Sons, 2017. 720p.

GIAA, Geomatics Industry Association of America, 2002. DIN 18723 Specification for Theodolite Accuracy. Professional Surveyor Magazine, 2002.

GONÇALVES, E. M. Planejamento de rede de monitoramento voltada para o monitoramento de estruturas empregando-se LST (Laser Scanner Terrestre). Dissertação de mestrado. Universidade Federal do Paraná, Programa de Pós-Graduação em Ciências Geodésicas, Curitiba, 2018. 102p.

KLEIN, I. et al. Rede de referência municipal para estações livres: uma proposta de baixo custo e grande abrangência. Revista Brasileira de Cartografia, vol. 69, n. 3, 2017. pp. 519-532 
KUANG, E. P. Geodetic Network Analysis and Optimal Design: concepts and application. Michigan, USA: Ann Arbour Press Inc, 1996. 368p.

MIRANDA, F. D.; VEIGA, L. A. K. Método de ressecção aplicado na determinação de coordenadas no monitoramento de pontos. Anais do III Simpósio Brasileiro de Ciências Geodésicas e Tecnologias da Geoinformação, Recife, 2010. pp. 001-005.

OGUNDARE, J. O. Precision Surveying: The Principles and Geomatics Practice. $1^{\mathrm{a}}$ ed. Vancouver: John Wiley \& Sons, 2015. 648p.

SHULTS, R.; ROSHCHYN, O. Preliminary Determination of Spatial Geodetic Monitoring Accuracy for Free Station Method. Geodetski list, vol. 70, n. 4, 2016. pp. $355-370$

SIGUEL, A. R. Monitoramento da barragem da usina hidrelétrica Mauá utilizando irradiação tridimensional. Dissertação de Mestrado. Universidade Federal do Paraná; Programa de Pós-Graduação em Ciências Geodésicas, Curitiba, 2013. 171p.

SILVA, I.; SEGANTINI, PCL. Topografia para Engenharia - Teoria e Prática de Geomática. $1^{a}$ ed. Rio de Janeiro: Elsevier Editora Ltda, 2015. 412p. 